\title{
Prevalence and Correlates of Healthy Aging Among Elderly Aged 65 Years and Over - 6 PLADs, China, 2019
}

\author{
Zhaoxue Yin ${ }^{1}$; Xin $\mathrm{Gao}^{1}$; Xiaochang Zhang'; Xiang $\mathrm{Si}^{1}$; Xiaolei Zhu' ${ }^{1}$; Jixiang Ma $\mathrm{Ma}^{2, .}$
}

\begin{abstract}
Summary
What is already known on this topic?

Healthy aging among Chinese older people has low prevalence. Some sociodemographic and lifestyle factors were shown to be associated with healthy aging. What is added by this report?

The age-adjusted prevalence of healthy aging in the 6 provincial-level administrative divisions (PLADs) of China is $15.8 \%$ in 2019. County-level factors, such as the prevalence of healthy communities in a county, as well as some sociodemographic variables and physical exercise, are potential factors of healthy aging.

What are the implications for public health practice?

These findings showed that more targeted actions, including generalizing healthy communities and individual-level interventions, may be of great importance for healthy aging.
\end{abstract}

China's population is known to be aging, but research on the overall health of the aging population is limited. This study aimed to evaluate the prevalence of healthy aging as defined in Table 1 and its correlates among China's elderly aged 65 years and over through the community-based Healthy Aging Evaluation Longitudinal Study in China (HAELS), which was conducted in 6 provincial-level administrative divisions (PLADs) in 2019. Descriptive statistics and multilevel logistic regression were used. Results showed the ageadjusted prevalence of healthy aging in the 6 PLADs in 2019 was $15.8 \%$. The prevalence of communities that satisfied criteria of being a "healthy community" at the county-level was significantly associated with healthy aging [odds ratio (OR): 1.20 per $10 \%$ increase in prevalence; $95 \%$ confidence interval $(95 \% \mathrm{CI})$ : 1.05-1.38]. Age, level of education, marital status, personal wealth, and physical exercise were also potential factors influencing healthy aging $(P<0.05)$. More targeted interventions should be implemented to improve healthy aging.

The number of elderly people aged 65 years and over in China was 176 million in 2019, accounting for $12.6 \%$ of the population (1). Aging is a multifaceted process that affects physical, mental, and social functions. Rowe and Kahn have proposed a conceptual model of aging that describes aging as "usual" and "successful" (2); however, the "successful" category has been criticized due to potential stigmatization of the "usual" category as unsuccessful aging (3), so the term healthy aging is used in this paper. The Rowe and Kahn concept of successful aging has been widely used to measure healthy aging (4-5), and the specific measurements for the 5 criteria [no major disease, no disability, high cognitive function, high physical function, and active engagement with life (6)] used in this study are provided in Table 1.

HAELS was initiated in 2019 in 6 PLADs (Beijing, Shandong, Jilin, Jiangxi, Ningxia, and Guangxi) and collected high-quality information from representative samples of elderly individuals aged 65 years and over to evaluate and monitor the trends of healthy aging. These 6 PLADs were selected based on economic development level; 2 counties or city districts were randomly sampled from each province or municipality, respectively; 2 towns or street districts were sampled from each county or city district, respectively, using multistage stratified probability-proportional-to-size (PPS) sampling; and within each town or street district, 2 villages or communities, respectively, were selected using the PPS sampling method. In the final stage, within each village or community, 100 participants were randomly sampled from residents aged 65 years and over with consideration of the overall PLAD-based proportion of the 2 age groups (aged 65-79 years and $\geq 80$ years). Overall, HAELS recruited 4,800 participants and had a final sample size of 4,690 for a response rate of $97.7 \%$.

County-level information for the participants was also collected including the total population aged 65 years and over, the gross domestic product (GDP) per capita, the implementation of long-term care insurance, the total number of villages/communities, and the prevalence of communities that were deemed 
TABLE 1. Criteria used to define 'healthy aging' in the HAELS conducted in 6 PLADs of China in 2019.

\begin{tabular}{|c|c|c|}
\hline $\begin{array}{l}\text { Concept model of } \\
\text { Rowe and Kahn }\end{array}$ & Definition & Measurements in this study \\
\hline No major disease & $\begin{array}{l}\text { No major chronic disease and no depression } \\
\text { symptom }\end{array}$ & $\begin{array}{l}\text { None of the following chronic diseases: cancer, chronic lung } \\
\text { disease, heart disease, and stroke; } 15 \text {-item geriatric depression } \\
\text { scale }<5\end{array}$ \\
\hline No disability & No limitations in activities of daily living & $\begin{array}{l}\text { Reporting no difficulty in performing activities of bathing, } \\
\text { dressing, toileting, indoor transferring, continence, and feeding }\end{array}$ \\
\hline $\begin{array}{l}\text { High cognitive } \\
\text { function }\end{array}$ & Getting median or higher score using MMSE & MMSE $\geq 26$ scores \\
\hline High physical function & $\begin{array}{l}\text { High performance in the physical tests or reporting } \\
\text { no difficulty in moderate and high intense activities }\end{array}$ & $\begin{array}{l}\text { Time to complete } 4 \text { meter gait speed test: }<4.75 \text { seconds (in the } \\
\text { high tertile group); Time to complete the FTSST: }<11.34 \\
\text { seconds (in the high tertile group); Performing moderate and } \\
\text { high intense physical activities every week }\end{array}$ \\
\hline $\begin{array}{l}\text { Active social } \\
\text { engagement }\end{array}$ & $\begin{array}{l}\text { Reported involvement in those outdoor social } \\
\text { activity }\end{array}$ & $\begin{array}{l}\text { Reporting "joining in the social activities with friends or families } \\
\text { at least once per week in the last year," "doing physical exercise } \\
\text { outdoors," or "playing poker with friends" at least once per week }\end{array}$ \\
\hline
\end{tabular}

"healthy". Healthy communities are part of the "China Healthy Lifestyle for All” national campaign ( 7 ), which promotes the construction of communities that have clean environments, places for physical activity and exercise, trails, "health huts", and activities promoting healthier lifestyles. The prevalence of healthy community was defined as the proportion of healthy communities/villages to the total number of communities/villages in one county. The HAELS study was approved by the ethics committee of Chinese Center for Disease Control and Prevention, and all participants (or proxies) provided written informed consent.

Descriptive statistics were performed to show the prevalence and $95 \% \mathrm{CI}$ of healthy aging. The ageadjusted prevalence and the corresponding 95\% CI were also calculated according to the proportion of the two age groups among the elderly in the 6 PLADs. Considering the hierarchical structure of the data and the dichotomous nature of healthy aging, two-level random intercept logistic regressions were applied using the programs of PROC GLIMMIX and PROC NLMIXED; the OR and 95\% CI for healthy aging were estimated. First, the null model was fit with no independent variables included to determine whether the data in this study was sufficient to assess the random effect at the second level (county-level) or not; then three models were further fit: model 1 , only level 2 explanatory variables including residence, GDP per capita, long-term care insurance, and prevalence of healthy community in a county were included; in model 2, level 2 explanatory variables and sociodemographic variables (level 1 explanatory variables) were included; and in model 3 , lifestyles, as level 1 explanatory variables, were further included. All statistical analyses were performed with SAS (version
9.4, SAS Institute Inc., Cary, NC, US). $P<0.05$ was considered statistically significant; all $P$ values were two-sided.

As shown in Table 2, the crude prevalence of healthy aging was $16.9 \%$ among older people in the 6 PLADs, and the age-adjusted prevalence was $15.8 \%$. The ageadjusted rate of "no major diseases", "no disability", "high cognitive function", "high physical function", and "active engagement with life" was $43.0 \%, 88.7 \%$, $49.1 \%, 79.3 \%$, and $69.0 \%$, respectively.

The empty model showed there was significant variation in the effect of healthy aging across counties $(P=0.04)$, and the intra-class correlation coefficient in the null model was $7.5 \%(P=0.03)$, which meant the random effect model that considering the effect of clustering should be fit. As shown in Table 3, the prevalence of healthy communities as a level-2 predictor was positively associated with healthy aging with $\mathrm{OR}=1.20$ (95\% CI: 1.05-1.38), which meant that odds of healthy aging would increase $20 \%$ per $10 \%$ increases in the prevalence of healthy communities in a county-level region. Age was negatively associated with healthy aging, while high education level, being married, having high wealth, and doing physical exercise were potential protective factors of healthy aging $(P<0.05)$.

\section{DISCUSSION}

This study demonstrated that the age-adjusted prevalence of healthy aging was $15.8 \%$ among the older people aged 65 years and over in 6 PLADs. The proportion of healthy communities in a county/district, as well as some demographic variables and physical exercise were factors affecting healthy aging. 


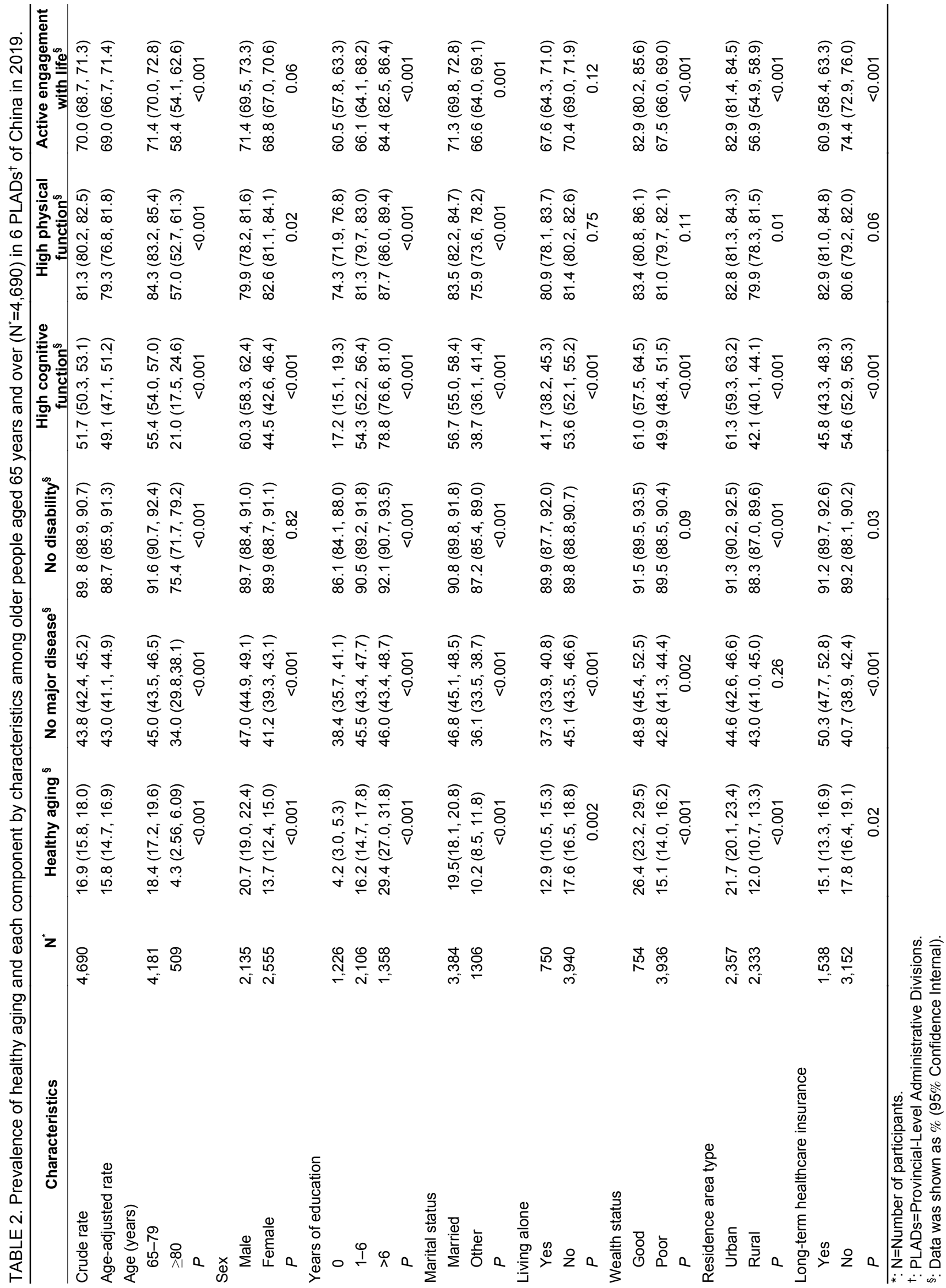


TABLE 3. Multilevel logistic regression of healthy aging among elderly people aged 65 years and over $\left(N^{*}=4,690\right)$ in 6 $\mathrm{PLADs}^{\dagger}$ in China in $2019^{\S}$.

\begin{tabular}{|c|c|c|c|}
\hline Variables & Model 1 & Model 2 & Model 3 \\
\hline \multicolumn{4}{|l|}{ County-level variables } \\
\hline Rural (ref.=urban) & $0.55(0.38,0.78)^{\pi}$ & $0.80(0.55,1.16)$ & $1.05(0.68,1.62)$ \\
\hline GDP per capital (per 1,000 dollars) & $1.00(0.97,1.03)$ & $0.90(0.64,1.26)$ & $0.95(0.64,1.41)$ \\
\hline Long-term care policy (ref.=no) & $1.12(0.79,1.59)$ & $1.17(0.81,1.71)$ & $1.38(0.89,2.13)$ \\
\hline Prevalence of health community (per 10\% increase) & $1.21(1.09,1.35)^{\pi}$ & $1.23(1.10,1.39)^{\pi}$ & $1.20(1.05,1.38)^{* *}$ \\
\hline \multicolumn{4}{|l|}{ Sociodemographic variables } \\
\hline \multicolumn{4}{|l|}{ Age (ref. $=65-79$ years) } \\
\hline Age $\geq 80$ years & & $0.27(0.16,0.45)^{\pi}$ & $0.29(0.17,0.48)^{\pi}$ \\
\hline \multicolumn{4}{|l|}{ Sex (ref.=male) } \\
\hline Female & & $0.83(0.68,0.99)^{* *}$ & $0.85(0.68,1.06)$ \\
\hline \multicolumn{4}{|l|}{ Education years $($ ref. $=0$ ) } \\
\hline $1-6$ & & $3.90(2.73,5.58)^{\pi}$ & $3.82(2.66,5.53)^{\pi}$ \\
\hline$>6$ & & $6.49(4.43,9.47)^{\pi}$ & $6.11(4.13,8.98)^{\pi}$ \\
\hline \multicolumn{4}{|l|}{ Marital status (ref.=other status) } \\
\hline Married & & $1.43(1.08,1.90)^{* *}$ & $1.38(1.04,1.82)^{* *}$ \\
\hline \multicolumn{4}{|l|}{ Living alone (ref.=no) } \\
\hline Yes & & $1.16(0.84,1.59)$ & $1.15(0.83,1.58)$ \\
\hline \multicolumn{4}{|l|}{ Wealth status (ref.=poor) } \\
\hline Good & & $1.92(1.52,2.41)^{\pi}$ & $1.75(1.40,2.22)^{\pi}$ \\
\hline \multicolumn{4}{|l|}{ Lifestyle variables } \\
\hline Smoke & & & $0.96(0.76,1.19)$ \\
\hline Alcohol drinking & & & $1.15(0.91,1.44)$ \\
\hline Tea & & & $1.01(0.81,1.25)$ \\
\hline Physical exercise & & & $2.62(2.11,3.23)^{\pi}$ \\
\hline
\end{tabular}

*: N=Number of participants.

†: PLADs=Provincial-Level Administrative Divisions.

§: Data was shown as odds ratio (95\% confidence internal). The null model, with no independent variables included, was fit; results assessed the random effect at level 2 (county/city-district level) and the three models were then fit. Model 1: only level 2 explanatory variables, including residence, GDP per capita, and long-term healthcare insurance, were included. Model 2: level 2 explanatory variables and sociodemographic variables (level 1) were included. Model 3: smoking, alcohol drinking, tea, and physical activities were further included.

T: $P<0.01$

${ }^{* *}: P<0.05$.

The prevalence found in this study was slightly higher than the national prevalence reported by the China Health and Retirement Longitudinal Study (CHARLS) in China in 2011 (13.2\%) (6), and further analysis showed that the increase came mainly from the dimensions of physical function and social engagement. The prevalence found in this study was lower, however, than that of a comparable study conducted in Singapore in 2013 (19.6\%), and the difference was most likely related to the higher prevalence of 'active engagement with life' in Singapore (8). However, the results of this study showed a higher prevalence than the mean level of European countries
(8.5\%) from 2004 to 2007 (9). The discrepancy across studies is also likely related to the inconsistent measurements of components of healthy aging, especially the dimension of "high physical function" and "active engagement with life." However, there is currently no consensus on exactly what specific measurements should be included in the construct of healthy/successful aging.

In this study, the finding of negative association between age and healthy aging was not unexpected. Education was found to be positively associated with healthy aging, which was consistent with recent studies $(6,10)$. Those with higher levels of education may 
possess greater knowledge and skills that enable them to have good lifestyles, participate in their communities, and avoid chronic diseases. Good wealth status was found to be one potential protective factor of healthy aging, which has been reflected in previous studies $(4,8)$, and it is possible that older people with good wealth status can more easily access healthcare resources. Physical exercise was found to be positively associated with healthy aging, which was consistent with previous studies (11).

This study also found that the prevalence of healthy community was positively related with healthy aging, which was supported by a previous study that suggested that older adults living in communities with proper health-related infrastructure had higher odds of experiencing healthy aging (4). Constructing healthy communities that have health-promoting elements in the community could be beneficial to healthy aging, and environmental resources were also shown to be an important factor in explaining inequality in healthy aging (12).

The findings are subject to some limitations. First, causal association cannot be established because this data was cross-sectional. Second, elderly aged 80 and over were less sampled in this study, considering the proportion of this age group in the 6 PLADs. However, the prevalence was age-adjusted to address this challenge. Third, some variables, including genetic factors and interaction effect between variables, were not included in the model.

These findings have important public health implications. They showed that more targeted actions, including county-level as well as individual-level interventions, should be taken to improve healthy aging.

Fundings: National Key R\&D Program of China (2020YFC2003000), China CDC's NCD and tobacco control program (131031001000190010).

Acknowledgments: Field survey staff of HAELS.

doi: $10.46234 / \mathrm{ccdcw} 2021.019$

\#Corresponding author: Jixiang Ma, majix@163.com.
Division of non-communicable diseases and elderly health management, Chinese Center for Disease Control and Prevention, Beijing, China; ${ }^{2}$ Shandong Center for Disease Control and Prevention, Jinan, Shandong, China.

Submitted: December 18, 2020; Accepted: January 19, 2021

\section{REFERENCES}

1. National Bureau of Statistics of China. Statistical communiqué of the people's republic of China on the 2019 national economic and social development. http://www.stats.gov.cn/english/PressRelease/202002/t20 200228_1728917.html. [2020-2-28].

2. Rowe JW, Kahn RL. Successful aging. Gerontologist 1997;37(4):433 40. http://dx.doi.org/10.1093/geront/37.4.433.

3. Martinson M, Berridge C. Successful aging and its discontents: a systematic review of the social gerontology literature. Gerontologist 2015;55(1):58 - 69. http://dx.doi.org/10.1093/geront/gnu037.

4. McLaughlin SJ, Chen Y, Tham SSX, Zhang JA, Li LW. Healthy aging in china: benchmarks and socio-structural correlates. Res Aging 2020;42(1):23 - 33. http://dx.doi.org/10.1177/0164027519879105.

5. Assmann KE, Andreeva VA, Jeandel C, Hercberg S, Galan P, KesseGuyot E. Healthy aging 5 years after a period of daily supplementation with antioxidant nutrients: a post hoc analysis of the French randomized trial SU.VI.MAX. Am J Epidemiol 2015;182(8):694 704. http://dx.doi.org/10.1093/aje/kwv105.

6. Liu HQ, Byles JE, Xu XY, Zhang M, Wu XS, Hall JJ. Evaluation of successful aging among older people in China: results from China health and retirement longitudinal study. Geriatr Gerontol Int 2017;17(8):1183 - 90. http://dx.doi.org/10.1111/ggi.12848.

7. Yang YB, Wang JL, Shi WH, Ma JX. The trend analysis of healthy settings development for China Healthy Lifestyle for All from 2013 to 2018. Chin J Prev Control Chronic Dis 2019;27(10):732 - 5. http://dx.doi.org/10.16386/j.cjpccd.issn.1004-6194.2019.10.004. (In Chinese).

8. Subramaniam M, Abdin E, Vaingankar JA, Sambasivam R, Seow E, Picco L, et al. Successful ageing in Singapore: prevalence and correlates from a national survey of older adults. Singapore Med J 2019;60(1):22 - 30. http://dx.doi.org/10.11622/smedj.2018050.

9. Hank K. How "successful" do older Europeans age? Findings from SHARE. J Gerontol B Psychol Sci Soc Sci 2011;66B(2):230 - 6 . http://dx.doi.org/10.1093/geronb/gbq089.

10. Wu YT, Daskalopoulou C, Terrera GM, Niubo AS, Rodríguez-Artalejo $\mathrm{F}$, Ayuso-Mateos JL, et al. Education and wealth inequalities in healthy ageing in eight harmonised cohorts in the ATHLOS consortium: a population-based study. Lancet Public Health 2020;5(7):e386-94. http://dx.doi.org/10.1016/S2468-2667(20)30077-3.

11. Moreno-Agostino D, Daskalopoulou C, Wu YT, Koukounari A, Haro JM, Tyrovolas $S$, et al. The impact of physical activity on healthy ageing trajectories: evidence from eight cohort studies. Int J Behav Nutr Phys Act 2020;17(1):92. http://dx.doi.org/10.1186/s12966-020-00995-8.

12. Stephens C, Szabó Á, Allen J, Alpass F. A capabilities approach to unequal trajectories of healthy aging: the importance of the environment. J Aging Health 2019;31(9):1527 - 48. http://dx.doi.org/ $10.1177 / 0898264318779474$ 